\title{
Super-X EDS Characterization of Chemical Segregation within a Superlattice Extrinsic Stacking Fault of a Ni- based Superalloy
}

\author{
Robert E. A. Williams ${ }^{1,2}$, Tim Smith ${ }^{1}$, Bryan D. Esser ${ }^{1}$, Nikolas Antolin ${ }^{1}$, Wolfgang Windl ${ }^{1}$, David W. \\ McComb $^{1,2}$, Michael J. Mills ${ }^{1}$, Hamish L. Fraser ${ }^{1}$ \\ ${ }^{1}$ Department of Materials Science and Engineering, The Ohio State University, 477 Watts Hall, 2041 \\ College Road, Columbus, OH 43210, USA \\ ${ }^{2}$ Center for Electron Microscopy and Analysis, The Ohio State University, 1305 Kinnear Road, \\ Columbus, $\mathrm{OH} 43212$, USA
}

Superalloys are essential materials for high temperature applications in aerospace and energy production. Improving the temperature capability of disk alloys by a modest $25^{\circ} \mathrm{C}$ could translate into approximately a $1 \%$ increase in aircraft engine efficiency, resulting in significant cost savings as well as benefit environmental impact by reducing carbon emissions. While superalloys are prime candidates for an ICME approach to accelerated alloy development, at present this approach is severely hampered by the lack of quantitative models that connect alloying effects to deformation mechanisms. This linkage is critical for developing quantitative, physics-based deformation models, but has been limited severely by the complexity of the alloys (typically 10 components or more). One aspect, critical to a successful model is accurate determination of the degree of segregation to defects, such as faults, in order to enable the identification of the compositional configuration of such defects.

The examples presented in this work consist of stacking faults created by superpartial dislocation shearing of the $\gamma^{\prime}$ precipitates during high temperature $\left(760^{\circ} \mathrm{C}\right)$ creep in an advanced, polycrystalline $\mathrm{Ni}$ disk superalloy. Under the deformation conditions studied, conventional TEM defect analysis determined this as the primary mode of precipitate shearing and it is expected to be a critical, ratelimiting process. Recent analysis of these faults using a Super-X system without a probe corrector has revealed for the first time that there appears to be a chemical signature associated with these faults, as evidenced in Fig. 1(a)[1]. In this case, involving a two-layer, superlattice extrinsic stacking fault (SESF), Al appears to be deficient at the fault while the concentrations of $\mathrm{Ti}$ and $\mathrm{Ta}$ (and $\mathrm{Cr}$, not shown) are enhanced at the fault. The information exhibited in Fig. 1(a) appears to indicate elemental segregation at the fault, however, the compositional details are not resolved on an atomic scale, which is necessary to develop a detailed understanding of the configuration of these defects for inclusion in a predictive model of deformation.

The results shown in Fig. 1(b) were obtained using a DCOR probe corrected STEM and demonstrate remarkably the improved resolution for XEDS mapping; made possible with $5^{\text {th }}$ order probe aberration correction and the increased collection efficiency and solid angle of the Super-X collection system of a FEI Titan Themis ${ }^{\mathrm{TM}}$. Features that are now characterizable for the first time in these [110] zone axis maps are: (a) discriminating the predominantly $\mathrm{Ni}(001)$ planes from the "mixed" $\mathrm{Ni}$ and Al planes, which also contain Ta and Ti in the "bulk"; (b) a slight reduction in the Ni content at the two-layer fault, occurring primarily on "mixed" (001) plane; (c) Ta and Ti replaces Ni and Al on the mixed planes; (d) a rectangular grid of columns with high Ta concentration corresponds to the high intensity columns in the HAADF images. These structural and chemical characteristics are consistent with the $\eta$ phase, which can form in superalloys and is deleterious in bulk form[2]. Preliminary first principles DFT calculations 
indicate that a single unit cell width of $\eta$ phase having a composition estimated directly from the EDS data has a significantly lower energy than an SESF in the absence of segregation.

The work to be presented demonstrates that, while indications of elemental segregation may be gleaned from experiments performed on an uncorrected STEM equipped with Super-X EDS collection, the atomic-scale, elemental information provided by $5^{\text {th }}$ order probe aberration corrected STEM equipped with Super-X EDS has provided direct chemical information related to SESF's for the first time and provided crucial information towards more accurate modeling of segregation phenomena. Post processing and quantification of data will also be discussed, as this is a critical aspect of apparent, atomic-resolution XEDS data.

Refereneces:

[1] G. B. Viswanathan, et al., Scripta Materialia 94 (2015): p. 5-8.

[2] E. J. Pickering, et al., Acta Materialia, 60(6-7), (2012): 2757-2769.

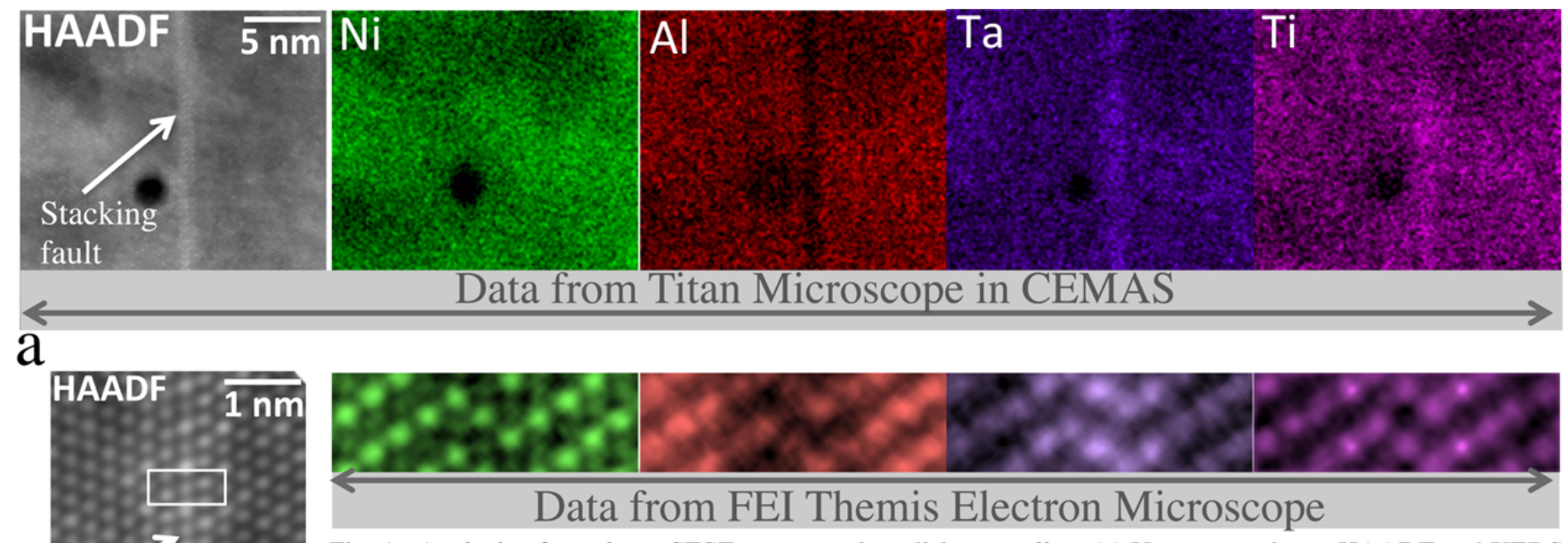

Fig. 1: Analysis of two-layer SESF structures in a disk superalloy. (a) Upper row shows HAADF and XEDS data obtained with the present Titan G3 with ChemiSTEM system, showing indication of Ta and Ti partitioning, and $\mathrm{Al}$ rejected, from the fault region. (b) Lower row shows HAADF and XEDS data obtained fault on a Themis electron microscope, showing atomic resolution composition maps (averaged) across the fault. 